\title{
Computing a Smart Device to Classify Recurring Outbreak of Copd ${ }^{\dagger}$
}

\author{
Bethanney Janney J. 1,*, Caroline Chriselda L. ${ }^{1}$, Chandana H. ${ }^{\text {, T. Tudhakar }}{ }^{1}$ \\ 1 Department of Biomedical Engineering Sathyabama Institute of Science and Technology, Chennai-119, India \\ * Correspondence: jannydoll@gmail.com; \\ $\dagger$ Presented at International e-Conference on Bioengineering for Health and Environment (ICBHE 2020)
}

Received: 5.07.2020; Revised: 10.07.2020; Accepted: 12.07.2020; Published: 15.07.2020

\begin{abstract}
Health care has experienced an unprecedented rise in the last decade. In order to keep their vital signs measured, patients will have to make regular visits to the doctor. The Cough detection device helps in the diagnosis and treatment of respiratory problems at an early age. Cough aims to remove mucus and bronchial diseases, which plays an important role in prevention and cure. More than 3 million individuals died of COPD in 2012, which is equivalent to 6 percent of all fatalities worldwide a year, according to world estimates. Citizens consider it impossible to track cough-related illnesses in rural areas because of the sophistication of the system and its testing costs. Nowadays, some people do have a mix of COPD and asthma. There is an immense demand for non-invasive ways of calculating these vital signs. This paper aims to develop and deploy a safe, inexpensive, low powered, nonintrusive, and effective device that can be worn daily and track the vital signs and show the performance to the mobile phone. The physician can also conveniently access such data through a wireless medium. This paper deals primarily with the acquisition of vital signs for signal conditioning and data: ECG, heart rate, blood pressure, body temperature, $\mathrm{Sp} 02$, cough rate, and respiration rate. This work proposes an innovative wearable cough detection method for early diagnosis and very early treatment of pulmonary diseases.
\end{abstract}

Keywords: Cough detection; Signal Conditioning; ECG; Respiration rate; SpO2 and Cough rate.

(C) 2020 by the authors. This article is an open-access article distributed under the terms and conditions of the Creative Commons Attribution (CC BY) license (https://creativecommons.org/licenses/by/4.0/).

\section{Funding}

This research received no external funding.

\section{Acknowledgments}

This research has no acknowledgment.

\section{Conflicts of Interest}

The authors declare no conflict of interest. 